\section{Echocardiographic Diagnosis of Right Aortic Arch with a Retroesophageal Segment and Left Descending Aorta}

\author{
DANIEL A. KVESELIS, MD \\ A. REBECCA SNIDER, MD \\ MACDONALD DICK II, MD \\ ALBERT P. ROCCHINI, MD
}

$\mathbf{T}$

wo-dimensional echocardiography has been used extensively to assess aortic arch anatomy in infants and children. Echocardiographic techniques for examination of left and right aortic arch, coarctation and interruption of the aorta, aortic aneurysm and double aortic arch have been well described. ${ }^{1.2}$ Right aortic arch with retroesophageal segment and left descending aorta, an unusual form of vascular ring, has been described using angiography, barium swallow and computed axial tomography. ${ }^{3-5}$ In this report, we describe 2-dimensional (2-D) echocardiographic findings in an infant with this unusual form of vascular ring.

A 7-month-old, 7.3-kg boy with the clinical diagnoses of CHARGE association, dextrocardia, pulmonary valve stenosis and recurrent pulmonary infections was evaluated by 2-D echocardiography, barium swallow and cardiac catheterization. The aortic arch was visualized by positioning the transducer in the

From the C.S. Mott Children's Hospital, F1125, Box 66, University of Michigan, Ann Arbor, Michigan 48109. Manuscript received June 24, 1985; revised manuscript received October 23, 1985, accepted Octobcr 25, 1085. suprasternal notch and orienting the plane of sound in the usual fashion for imaging a left aortic arch (i.e., with the plane of sound passing between the left scapular tip and right nipple). The aortic arch had a hairpin appearance suggestive of a persistent third arch. From the suprasternal short-axis view, the first brachiocephalic vessel coursed anteriorly, superiorly and leftward (Fig. 1). With posterior angulation of the transducer in the suprasternal short-axis view, the right common carotid and right subclavian arteries were imaged arising from the transverse arch. In the most posterior short-axis plane, a large segment of the arch passed behind the air-filled trachea and joined a left descending aorta. Subcostal short-axis views confirmed that the abdominal descending aorta was leftsided.

Cardiac catheterization showed mild pulmonary valve stenosis [right ventricle $52 / 6$, pulmonary artery 20/11 and aorta $100 / 70 \mathrm{~mm} \mathrm{Hg}$ ). Aortography revealed a persistent third right aortic arch with a retroesophageal segment and left descending aorta [Fig. 2). The left common carotid artery was the first brachiocephalic vessel arising from the aorta. These findings werc confirmed by barium swallow. The left-sided ligamentum arteriosus was surgically divided. Posterior tracheal compression was observed. Postoperative recovery was uneventful.

Right aortic arch with a retroesophageal segment occurs by regression of the segment between the origins of the left common carotid and left subclavian arteries. ${ }^{6}$ Echocardiographic diagnosis of this defect is based on several anatomic features. Because of the position of the retroesophageal segment and a left descending aorta, the entire aortic arch can be imaged in a plane typical for a left aortic arch, even though a right aortic arch is actually present. The diagnosis of a right aortic arch is suspected when the first brachiocephalic vessel courses leftward and superiorly. If the first brachiocephalic vessel is to the left, then the arch would

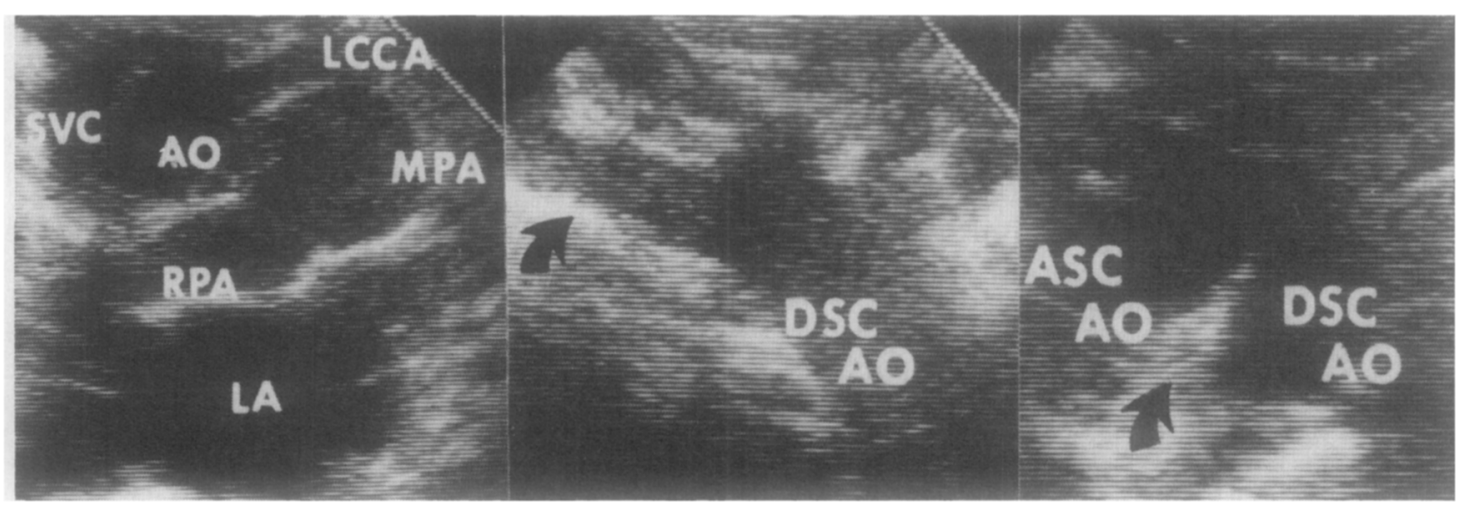

FIGURE 1. Left, suprasternal notch 2-dimensional echocardlogram (short axis) showing the ascending aorta (Ao) with the first branch arising from it being the left common carotid artery (LCCA). MIddle, sweeping toward the long-axis view, the retroesophageal segment (arrowhead) to the descending aorta (DscAo). Rlght, the long-axis vlew shows the hairpin appearance of the aortic arch as visualized from the usual left arch plane. The ascending aorta (AscAo) and descending aorta a well visualized with the right mainstem branches (arrowhead) beneath them. LA = left atrium; MPA = main pulmonary artery; RPA $=$ right pulmonary artery; SCV $=$ superior vena cava. 


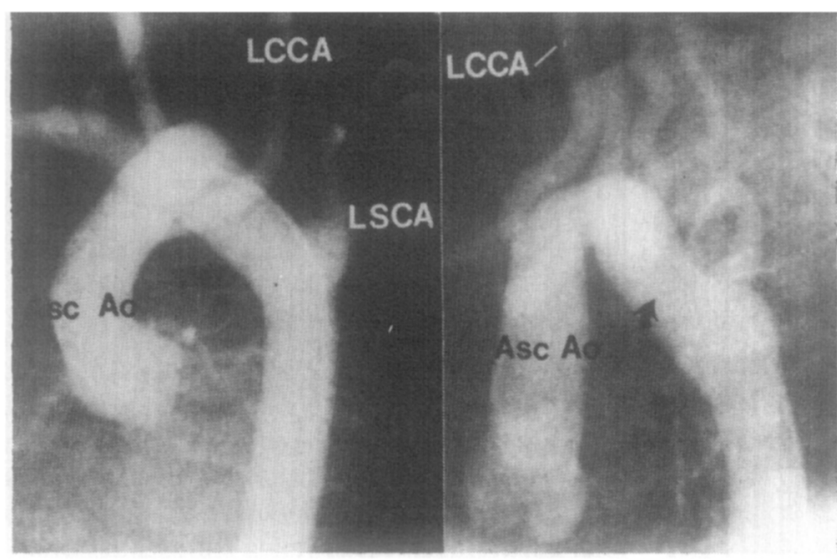

FIGURE 2. Aortic root angiogram in the posteroanterior (left) and lateral (right) projections. Note the sweeping of the aorta rightward, then posterior with the left common carotid artery (LCCA) arising as the first brachiocephalic vessel. Arrowhead indicates retroesophageal segment. AscAo = ascending aorta; LSCA $=$ left subclavlan artery. have to be right sided. Since this anomaly is often associated with a persistent third or cervical arch, an additional echocardiographic feature is a hairpin appearance of the arch.

\section{References}

1. Snider AR, Silverman NH. Suprasternal notch echocardiography: a twodimensional technique for evaluating congenital heart disease. Circulation 1981;63:165-173.

2. Celano V, Pieroni DR, Gingell RL, Roland JA. Two-dimensional echocardiographic recognition of the right aortic arch. Am J Cardiol 1983;51:15071512 .

3. Bennett EV Jr, Grover FL, Trinkle JK. Right aortic arch causing left bronchus obstruction. Texas Heart Inst I 1984;11:204-207.

4. Blieden LC, Schneeweiss A, Deutsch V, Neufeld HN. Right aortic arch with left descending aorta (circumflex aorta): roentgenographic diagnosis. Pediatr Radinl 1978:6:208-210.

5. Taber P, Chang LWM, Campion GM. Diagnosis of retro-esophageal right aortic arch by computed tomography. I Comput Assist Tomogr 1979;3:684685.

6. Edwards JE. Congenital cardiovascular causes of tracheobronchial and/or esophageal obstruction. In: Tucker BL, Lindesmith GG, eds. First Clinical Conference on Congenital Heart Disease. New York: Grune \& Stratton, 1979:66-69.

\section{Massive Tricuspid Regurgitation Caused by Intramyocardial Hydatid Cyst}

\author{
FRANCISCO GARCIA-FERNANDEZ, MD \\ ESTEBAN LÓPEZ DE SÁ, MD \\ CARLOS GAMALLO, MD \\ CARLOS PAVÖN, MD \\ JOSÉ SOTILLO, MD
}

F

ewer than $2 \%$ of patients with hydatid disease have cardiac involvement. ${ }^{1}$ In $65 \%$ of those with cardiac involvement the cyst affects only the heart, usually the left ventricle. ${ }^{1,2}$ We present a case of massive tricuspid regurgitation due to a hydatid cyst in the ventricular septum, the first such case reported.

A 46-year-old man was admitted to the hospital for anasarca secondary to right-sided congestive heart failure. He was living in a rural environment. Several years before he had effort syncope, with generalized cutaneus flushing, from which he recovered spontaneously. At age 41 years hydatid disease was diagnosed after an episode of cough with elimination of hydatid cyst fragments in sputum. Thoracotomy was performed for removal of a large hydatid cyst in right lung. After this episode, he was in functional class II (New York Heart Association) with digitalis and diuretic therapy. He had tachypnea, central and pe-

From the Servicio de Cardiologia, Departamento de Anatomia Patológica, and Servicio de Cirugía Cardiaca, Hospital "La Paz," 28046 Madrid, Spain. Manuscript received November 21, 1985; revised manuscript received December 24,1985 , accepted December $26,1985$. ripheral cyanosis, jugular venous pressure of $22 \mathrm{~mm}$ $\mathrm{Hg}$ at $90^{\circ}$, a pansystolic murmur in the tricuspid area that increased with inspiration, a third heart sound, wheezing and fine rales in both pulmonary bases. $\mathrm{He}$ also had a large and painful liver, edema in the lower extremities up to the knees and clubbing of fingers and toes.

An electrocardiogram showed atrial fibrillation alternating with sinus rhythm, right ventricular hypertrophy and right bundle branch block. A chest x-ray showed enlargement of all chambers and multiple nodulary images in the lung fields suggestive of hydatid emboli. Hydatid serology titer was $1 / 5120$. Mmode and 2-dimensional echocardiograms showed a cystic mass in the ventricular septum with a free portion that involved the tricuspid valve (Fig. 1). The right-sided cardiac chambers were very dilated; images of regurgitation contrast echoes were present in the inferior cava vein during systole. Pulmonary artery pressure was $75 / 45 \mathrm{~mm} \mathrm{Hg}$. Right ventriculography showed massive tricuspid regurgitation and a motile intracavitary filling defect. Coronary arteriographic findings were normal. At surgery a large septal cyst was removed (Fig. 1). The tricuspid valve was replaced with a bioprothesis. The surgical frugment was composed of right septal wall tissue and 2 cysts (supravalvular and infravalvular of $4 \times 1.5 \times 1.5 \mathrm{~cm}$ and $3 \times 2.5 \times 2.5 \mathrm{~cm}$, respectively]. Both consisted of hydatid cyst with signs of necrosis and acute and chronic inflammation with granulomatous reaction and foreign body giant cells. After surgery, the patient had anuric acute renal failure that required peritoneal dialysis and several episodes of paralytic ileus. Pseudomona Aeruginosa pneumonia developed and the patient died 20 days after surgery.

The clinical picture of hydatid disease depends on cyst localization and size; the cyst may provoke angina 